Draft version December 20, 2019

Typeset using LATEX twocolumn style in AASTeX61

\title{
REVEALING THE CO X-FACTOR IN DARK MOLECULAR GAS THROUGH SENSITIVE ALMA ABSORPTION OBSERVATIONS
}

\author{
Gan Luo,${ }^{1,2}$ Di Li,${ }^{1,2,3}$ Ningyu Tang, ${ }^{1}$ J. R. Dawson, ${ }^{4}$ John M. Dickey, ${ }^{5}$ L. Bronfman, ${ }^{6}$ Sheng-Li Qin, ${ }^{7}$ \\ Steven J. Gibson,${ }^{8}$ Richard Plambeck, ${ }^{9}$ Ricardo Finger, ${ }^{6}$ Anne Green, ${ }^{10}$ Diego Mardones, ${ }^{11}$ Bon-Chul Koo, ${ }^{12}$ \\ AND NADIA LO ${ }^{6}$ \\ ${ }^{1}$ CAS Key Laboratory of FAST, National Astronomical Observatories, Chinese Academy of Sciences, Beijing 100101, China; \\ luogan@nao.cas.cn,dili@nao.cas.cn,nytang@nao.cas.cn \\ ${ }^{2}$ University of Chinese Academy of Sciences, Beijing 100049, China \\ ${ }^{3}$ NAOC-UKZN Computational Astrophysics Centre, University of KwaZulu-Natal, Durban 4000, South Africa \\ ${ }^{4}$ Department of Physics and Astronomy and MQ Research Centre in Astronomy, Astrophysics and Astrophotonics, Macquarie University, \\ NSW 2109, Australia \\ ${ }^{5}$ University of Tasmania, School of Maths and Physics, Hobart, TAS 7001, Australia \\ ${ }^{6}$ Astronomy Department, Universidad de Chile, Casilla 36-D, Santiago, Chile \\ ${ }^{7}$ Department of Astronomy, Yunnan University, and Key Laboratory of Astroparticle Physics of Yunnan Province, Kunming, 650091, \\ China \\ ${ }^{8}$ Western Kentucky University, Dept. of Physics and Astronomy, 1906 College Heights Boulevard, Bowling Green, KY 42101, USA \\ ${ }^{9}$ Radio Astronomy Laboratory, University of California, Berkeley, CA 94720 \\ ${ }^{10}$ Sydney Institute for Astronomy (SIfA), School of Physics, University of Sydney, NSW 2006, Australia \\ ${ }^{11}$ Departamento de Astronomía, Universidad de Chile, Casilla 36, Santiago de Chile, Chile \\ ${ }^{12}$ Department of Physics and Astronomy, Seoul National University Seoul 151-747, Korea
}

\section{Submitted to ApJL}

\begin{abstract}
Carbon-bearing molecules, particularly $\mathrm{CO}$, have been widely used as tracers of molecular gas in the interstellar medium (ISM). In this work, we aim to study the properties of molecules in diffuse, cold environments, where CO tends to be under-abundant and/or sub-thermally excited. We performed one of the most sensitive (down to $\tau_{\text {rms }}^{\mathrm{CO}} \sim 0.002$ and $\left.\tau_{\text {rms }}^{\mathrm{HCO}^{+}} \sim 0.0008\right)$ sub-millimeter molecular absorption line observations towards 13 continuum sources with the ALMA. CO absorption was detected in diffuse ISM down to $\mathrm{A}_{\mathrm{v}}<0.32 \mathrm{mag}$ and $\mathrm{HCO}^{+}$was down to $\mathrm{A}_{\mathrm{v}}<0.2 \mathrm{mag}$, where atomic gas and dark molecular gas (DMG) starts to dominate. Multiple transitions measured in absorption toward 3C454.3 allow for a direct determination of excitation temperatures $\mathrm{T}_{\mathrm{ex}}$ of $4.1 \mathrm{~K}$ and $2.7 \mathrm{~K}$, for $\mathrm{CO}$ and for $\mathrm{HCO}^{+}$, respectively, which are close to the cosmic microwave background (CMB) and provide explanation for their being undercounted in emission surveys. A stronger linear correlation was found between $\mathrm{N}_{\mathrm{HCO}}+$ and $\mathrm{N}_{\mathrm{H}_{2}}$ (Pearson correlation coefficient $\mathrm{P} \sim 0.93)$ than that of $\mathrm{N}_{\mathrm{CO}}$ and $\mathrm{N}_{\mathrm{H}_{2}}(\mathrm{P} \sim 0.33)$, suggesting $\mathrm{HCO}^{+}$being a better tracer of $\mathrm{H}_{2}$ than $\mathrm{CO}$ in diffuse gas. The derived $\mathrm{CO}$-to- $\mathrm{H}_{2}$ conversion factor (the $\mathrm{CO}$ X-factor) of $(14 \pm 3) \times 10^{20} \mathrm{~cm}^{-2}(\mathrm{~K}$ $\left.\mathrm{km} \mathrm{s}^{-1}\right)^{-1}$ is approximately 6 times larger than the average value found in the Milky Way.
\end{abstract}

Keywords: ISM: molecules - ISM: clouds - ISM: abundance 


\section{INTRODUCTION}

Hydrogen is the most abundant element in the universe and plays a crucial role in the chemical evolution of cosmic materials. In contrast to the $21 \mathrm{~cm}$ hyperfine transition of atomic hydrogen, molecular hydrogen has no easily accessible transitions (due to a lack of permanent dipole) in the cold $(\mathrm{T} \sim 15 \mathrm{~K})$ ISM, where $\mathrm{H}_{2}$ is the dominant component of gases. $\mathrm{CO}$ is the de-facto stand-in for $\mathrm{H}_{2}$ in both the Milky Way and external galaxies, ever since its discovery (Wilson et al. 1970). Total molecular gas is often estimated through an empirical CO-to- $\mathrm{H}_{2}$ conversion factor (hereafter, the $\mathrm{CO} \mathrm{X}$ factor), namely, $\mathrm{N}_{\mathrm{H}_{2}}=\mathrm{X}_{\mathrm{CO}} \mathrm{W}_{\mathrm{CO}}$, a typical mean value of which is $2 \times 10^{20} \mathrm{~cm}^{-2}\left(\mathrm{~K} \mathrm{~km} \mathrm{~s}^{-1}\right)^{-1}$ for the Milky Way (Bolatto et al. 2013).

However, $\mathrm{CO}$ is not always a reliable tracer of $\mathrm{H}_{2}$. A significant fraction of interstellar molecular gas is not traced by $\mathrm{CO}$ emission surveys (e.g. Dame et al. 2001) and is often referred to as dark molecular gas (DMG). DMG has been revealed by gamma-ray observations from the Energetic Gamma Ray Experiment Telescope (EGRET, Grenier et al. 2005) and Fermi (Remy et al. 2017), dust observations with Planck (Planck Collaboration et al. 2011), and $\mathrm{C}^{+}$observations from Galactic observations of TeraHertz C+ (GOTC+) survey (Pineda et al. 2013; Langer et al. 2014). CO emission fails to trace DMG in two critical aspects, abundance and excitation. The selfshielding of $\mathrm{H}_{2}$ can be achieved at much lower extinction than that for $\mathrm{CO}$. In regions with intermediate extinctions $\left(\mathrm{A}_{\mathrm{V}} \sim 0.2--2 \mathrm{mag}\right.$ see Li et al. 2018), ultraviolet dissociation significantly reduces the $\mathrm{CO}$ abundance from its cannonical value of $[\mathrm{CO}] /\left[\mathrm{H}_{2}\right]=10^{-4}$ (van Dishoeck \& Black 1988; Wolfire et al. 2010; Draine 2011). In such regions, the gas density can be lower than the critical density, making transitions such as CO (1-0) sub-thermally excited, further hampering the usability of $\mathrm{CO}$ emission as a $\mathrm{H}_{2}$ tracer.

The "missing" CO emission in diffuse and translucent clouds may lead to a higher CO X-factor than in dense molecular gas. Observational evidence from dust extinction and $\mathrm{CO}$ emission has revealed a larger $\mathrm{CO} \mathrm{X}$ factor in diffuse regions $\left(A_{v}<3\right.$ mag $)$ than in dense gas (Pineda et al. 2010) in the Taurus molecular cloud. A similar trend has also been found by Remy et al. (2017) toward six nearby anti-centre clouds. However, other studies have reported a mean value of the $\mathrm{CO} \mathrm{X}$-factor that is the same in diffuse and dense molecular clouds (Liszt et al. 2010; Liszt 2012), although there are considerable variations between individual sightlines.

Given the high temperatures of background sources, quasar absorption can be a much more sensitive and less biased probe of cold molecular gas, particularly, the DMG in the intermediate extinction regions. Absorption toward continuum sources has been commonly used to quantify the physical properties of the Galactic interstellar medium (e.g. Heiles \& Troland 2003; Dickey 2013; Li et al. 2018; Nguyen et al. 2018; Riquelme et al. 2018). In such absorption programs, it is possible to measure the column density accurately even when a transition is sub-thermally excited, to detect diffuse gas with lower column density, and to directly measure optical depth. Furthermore, absorption from multiple transitions facilitate an unambiguous determination of excitation temperature and column density simultaneously.

In addition to $\mathrm{CO}, \mathrm{HCO}^{+}$is a key reactant of $\mathrm{CO}$ formation, and has been shown to trace $\mathrm{H}_{2}$ well (Liszt \& Lucas 1998). The emission of $\mathrm{HCO}^{+}$is rare and weak in diffuse clouds (tens of $\mathrm{mK}$ in brightness temperature, Lucas \& Liszt 1996), and excitation temperatures derived from the two lowest transitions are comparable to the CMB (2.7-3.0 K, Godard et al. 2010), making absorption toward strong continuum sources a preferred probe of $\mathrm{HCO}^{+}$as well.

We have taken deep absorption observations of $\mathrm{CO}$ and $\mathrm{HCO}^{+}$toward 13 millimeter-wavelength point source calibrators using the ALMA. We present our observations and archival data in Section 2, and analyze the absorption profiles of the observed targets in Section 3. We derive the abundance of $\mathrm{CO}$ and $\mathrm{HCO}^{+}$and discuss the advantage of $\mathrm{HCO}^{+}$as a tracer of molecular gas in Section 5. Analysis of the CO X-factor is presented in Section 6. Our main results and conclusions are presented in Section 7.

\section{OBSERVATIONS AND DATA}

We have selected 13 calibration sources (3C454.3, 3C120, B0420-014, B0607-157, B0627-199, B1730130, B1741-038, B1742-078, B1933-400, B1954-388, B2223-052, B2227-088, and B2243-123) used by worldwide mm-wave arrays. In addition, none of these sightlines has CO emission in the CO survey in Dame et al. 2001. The sightlines of these sources lie in DMG (Planck Collaboration et al. 2011, $\mathrm{A}_{\mathrm{v}}: 0.37 \mathrm{mag} \sim 2.5 \mathrm{mag}$ ) or DMG-threshold (as defined in Li et al. 2018) regions, enabling us to explore the physical properties in DMG sightlines.

\subsection{Molecular lines}

We observed $\mathrm{CO}(1-0)$ and $\mathrm{HCO}^{+}(1-0)$ spectra toward 13 strong continuum sources with ALMA during 2015. We also observed two higher energy transitions, $\mathrm{CO}(2-1)$ and $\mathrm{HCO}^{+}(3-2)$, toward $3 \mathrm{C} 454.3$ to constrain the physical properties (see Section 4). The 
frequency resolution of the $\mathrm{CO}(1-0)$ and $\mathrm{HCO}^{+}(1-$ 0) lines is $61.035 \mathrm{KHz}$, which corresponds to velocity resolutions of $0.21 \mathrm{~km} \mathrm{~s}^{-1}$ at $89 \mathrm{GHz}$ and $0.18 \mathrm{~km} \mathrm{~s}^{-1}$ at $113 \mathrm{GHz}$. The frequency resolution of the $\mathrm{CO}(2-1)$ and $\mathrm{HCO}^{+}(3-2)$ lines is $122.070 \mathrm{KHz}$, which corresponds to velocity resolutions of $0.159 \mathrm{~km} \mathrm{~s}^{-1}$ at $230 \mathrm{GHz}$ and $0.137 \mathrm{~km} \mathrm{~s}^{-1}$ at $267 \mathrm{GHz}$. The continuum data covers a $1.8 \mathrm{GHz}$ bandwidth with a frequency resolution of 31 $\mathrm{MHz}$. The calibration of the raw data was performed using CASA (Common Astronomy Software Applications) (McMullin et al. 2007). Both calibrated continuum and spectral images were deconvolved with Briggs weighting using the Clean algorithm.

\subsection{Archival reddening data}

We use the E(B-V) map of Green et al. (2018, 2019) to estimate total gas column density. The $\mathrm{E}(\mathrm{B}-\mathrm{V})$ map is derived by combining high-quality stellar photometry of 800 million stars from Pan-STARRS 1, 200 million stars from 2MASS, and parallaxes of 500 million stars from Gaia (Green et al. 2019). It covers three-quarters of the whole sky at a spatial resolution of $3.4^{\prime}$ to $13.7^{\prime}$.

\subsection{Archival H I data}

We adopted the corrected HI column density derived from $\mathrm{ON}-\mathrm{OFF}$ observations in the millennium survey for 3C454.3 and 3C120 (Heiles \& Troland 2003; Nguyen et al. 2018). These H I spectra have a velocity resolution of $0.161 \mathrm{kms}^{-1}$ and an RMS noise of $56 \mathrm{mK}$ per channel. HI data for other sources are taken from the Parkes Galactic All Sky Survey (GASS) (McClure-Griffiths et al. 2009; Kalberla et al. 2010; Kalberla \& Haud 2015), in which the column density of $\mathrm{HI}$ is calculated by integration over a velocity range of $-400<\mathrm{V}<400 \mathrm{~km} \mathrm{~s}^{-1}$ under the assumption of $\tau \ll 1$, and is therefore a lower limit. The GASS data have an angular resolution of $16^{\prime}$, a spectral resolution of $0.82 \mathrm{~km} \mathrm{~s}^{-1}$ and an RMS noise of $57 \mathrm{mK}$ per channel. In addition, $\mathrm{HI}$ data for two bandpass calibrators $(2148+0657$ and 2232+1143; see Section 3.1) are taken from the Effelsberg-Bonn HI Survey (EBHIS), which has an angular resolution of $\sim 11^{\prime}$, a spectral resolution of $1.29 \mathrm{~km} \mathrm{~s}^{-1}$ and an RMS noise of $90 \mathrm{mK}$ per channel (Winkel et al. 2016).

\section{ABSORPTION PROFILES}

Absorption profiles can provide a direct measurement of optical depth, provided that the background source is sufficiently bright. Optical depth can tell us the column density along a sightline, with the proper assumption of the excitation conditions. In this section, we describe the extraction of absorption spectra and the fitting process.

\subsection{Detections and self-calibration}

Since our observing targets are strong millimeterwavelength continuum point sources, we can perform calibration using the sources themselves (selfcalibration). Eliminating separate calibrator observations would have cut our observing time by more than half. However, ALMA Phase 2 observations did not support this mode. Thus, we performed our calibration using the standard ALMA pipeline, which uses separate bandpass, phase, and flux calibrators.

Both $\mathrm{CO}(1-0)$ and $\mathrm{HCO}^{+}(1-0)$ absorption lines were detected toward 6 of 13 sources: 3C454.3, 3C120, 0607-157, 1730-130, 1741-038, and 1742-078. The two higher energy transitions - $\mathrm{CO}(2-1)$ and $\mathrm{HCO}^{+}(3-2)$ - observed toward 3C454.3 were also detected. In our $\mathrm{HCO}^{+}(1-0)$ absorption profiles, we found two emission components toward 3C120 and 3C454.3 (see Figure 1 (a) as an example). High brightness temperatures of $108 \mathrm{~K}$ (for $3 \mathrm{C} 120$ ) and $537 \mathrm{~K}$ (for 3C454.3), make it hard to believe that the emission could be excited by high-temperature gas, and suggest contamination from a bandpass calibrator. Thus, we performed self-calibration on 3C120 and 3C454.3, as well as their calibrators. As suspected, $\mathrm{HCO}^{+}(1-0)$ absorption was detected towards two bandpass calibrators $(2232+1143$ and 2148+0657), confirming that the "emission" components above are indeed contamination. The selfcalibrated and normally-calibrated spectra for 3C454.3 can be seen in Figure 1 (a). Data reduction toward 3C454.3, 3C120, (and also 2148+0657 and 2232+1143, which were added to our sample) was therefore conducted by self-calibration. Calibration toward all the other sources was conducted by normal calibration.

\subsection{Gaussian fitting of absorption profiles}

According to the radiative transfer equation:

$$
T_{R}^{*}=J_{v}\left(T_{b g}\right) e^{-\tau_{v}}+J_{v}\left(T_{e x}\right)\left(1-e^{-\tau_{v}}\right),
$$

where $\mathrm{T}_{\mathrm{R}}^{*}$ is the main beam brightness temperature, $\tau_{v}$ is the optical depth, and $\mathrm{J}_{v}(\mathrm{~T})=(\mathrm{h} v / \mathrm{k}) /(\exp (\mathrm{h} v / \mathrm{kT})-1)$. We can then express $\mathrm{e}^{-\tau_{v}}$ as

$$
e^{-\tau_{v}}=\frac{T_{R}^{*}-J_{v}\left(T_{e x}\right)}{J_{v}\left(T_{b g}\right)-J_{v}\left(T_{e x}\right)} .
$$

When the background continuum source is strong enough (e.g., 3C454.3 with $\left.\mathrm{J}_{v}\left(\mathrm{~T}_{\mathrm{bg}}\right) \sim 537 \mathrm{~K}\right)$, term $\mathrm{J}_{v}\left(\mathrm{~T}_{\mathrm{bg}}\right) \gg \mathrm{J}_{v}\left(\mathrm{~T}_{\mathrm{ex}}\right)\left(1-\mathrm{e}^{-\tau_{v}}\right)$, and equation 2 can be rewritten as

$$
e^{-\tau_{v}} \simeq \frac{T_{R}^{*}}{J_{v}\left(T_{b g}\right)} .
$$




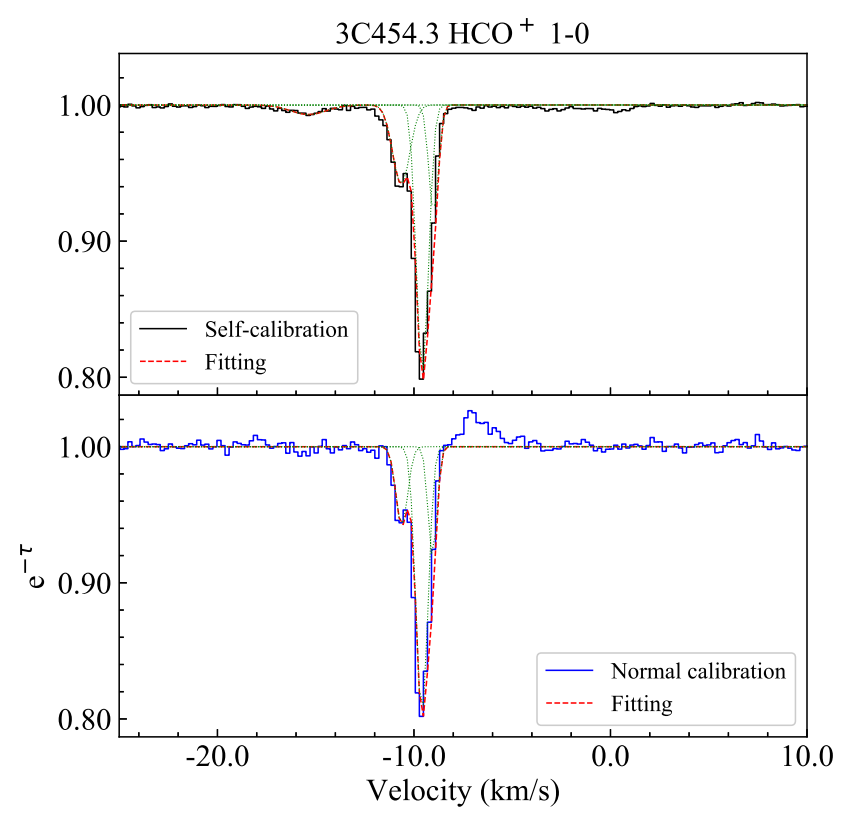

(a)

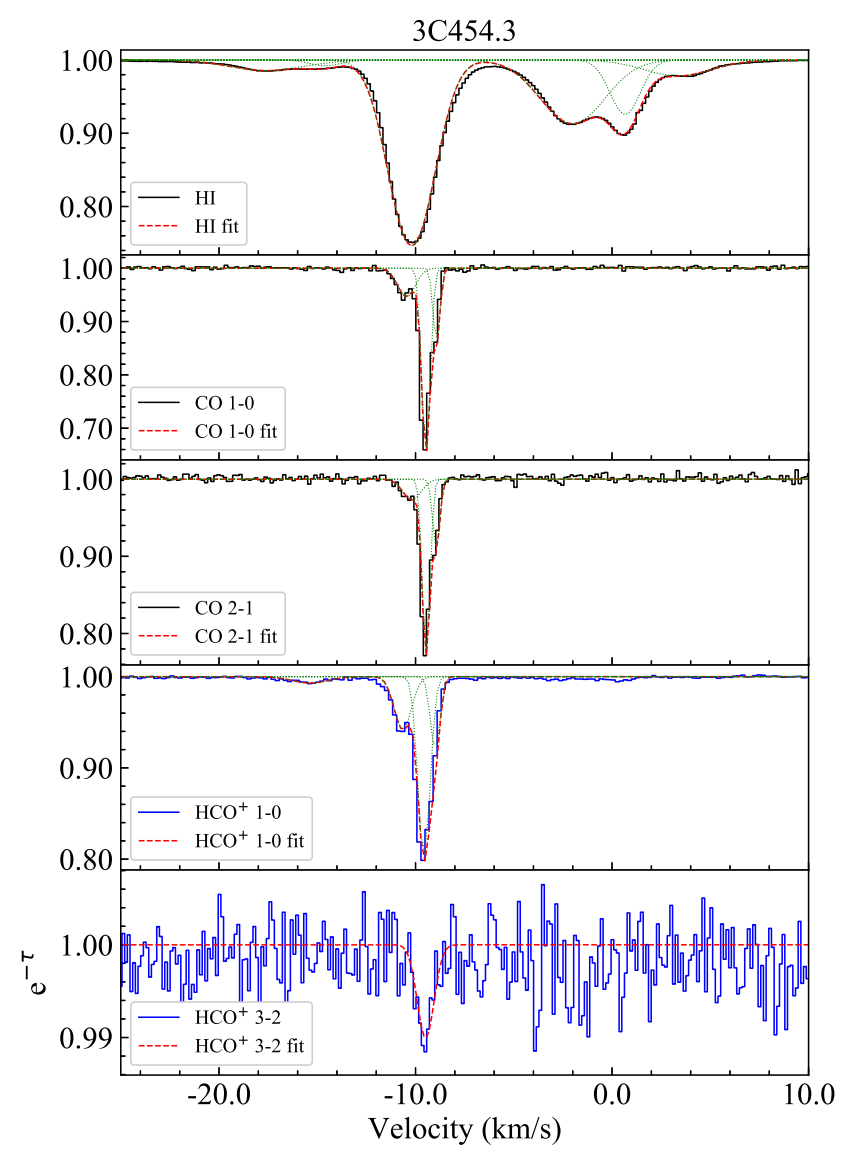

(b)

Figure 1. (a): Comparison of absorption spectra obtained through self- (black curve) and normal (blue curve) calibration. (b): The spectra of $\mathrm{HI}, \mathrm{CO}$ and $\mathrm{HCO}^{+}$(solid line) towards 3C454.3. The red dashed lines show the Gaussian fit profiles for each line, and the green dotted lines show each of the Gaussian components. The $\mathrm{x}$-axis and y-axis denote the velocity range in $\mathrm{km} \mathrm{s}^{-1}$ and the normalized line intensity, respectively.

The $\tau_{v}$ of each velocity component was derived by fitting the $\mathrm{e}^{-\tau_{v}}$ profiles with multiple Gaussian components. Equation 3 is only used for obtaining the $\tau_{v}$ value of 3C454.3. The other sources observed in this study have background brightness temperatures between $20 \mathrm{~K}$ to $176 \mathrm{~K}$, meaning that we must revert to equation 2 to compute $\mathrm{e}^{-\tau_{v}}$ profiles. For these, we adopt representative values of $\mathrm{T}_{\mathrm{ex}}$ computed for $3 \mathrm{C} 454$ (4.1 $\mathrm{K}$ for $\mathrm{CO}$ and $2.7 \mathrm{~K}_{\text {for }} \mathrm{HCO}^{+}$in Section 4$)$. The $\mathrm{e}^{-\tau_{v}}$ profiles of 3C454.3 are shown in Figure 1 (b).

There are twelve CO (1-0) components and twentythree $\mathrm{HCO}^{+}(1-0)$ components in our sample. Each $\mathrm{CO}$ (1-0) velocity component has a corresponding $\mathrm{HCO}^{+}$ (1-0) component. Those sightlines with CO detection suggesting that $\mathrm{CO}$ has been formed in diffuse gas down to $\mathrm{A}_{\mathrm{v}}$ value of $0.32 \mathrm{mag}$ (converting from $\mathrm{E}(\mathrm{B}-\mathrm{V})$ in Table 1 using $\left.A_{v} / E(B-V)=3.1\right)$. Eleven $\mathrm{HCO}^{+}$components have no $\mathrm{CO}$ detections, suggesting that these components lie in diffuse molecular gas where CO cannot form and that they may lie at the edge of the molecular cloud. Specifically, there are two sightlines without $\mathrm{CO}$ detections but which are rich in $\mathrm{HCO}^{+}$, indicating that $\mathrm{HCO}^{+}$exists down to $\mathrm{A}_{\mathrm{v}}$ value of $0.19 \mathrm{mag}$.

The velocity of these absorption profiles varies from $\sim-10-10 \mathrm{~km} \mathrm{~s}^{-1}$, indicating that the cloud components along the sightline are nearby clouds. The optical depths of $\mathrm{CO}(1-0)$ and $\mathrm{HCO}^{+}(1-0)$ are in the range of $\sim 0.04-$ 1.55 and $\sim 0.007-0.98$, respectively. The linewidths of $\mathrm{HCO}^{+}\left(0.56-3.96 \mathrm{~km} \mathrm{~s}^{-1}\right)$ are sightly wider than $\mathrm{CO}$ 
$\left(0.21-3.79 \mathrm{~km} \mathrm{~s}^{-1}\right)$ by a factor of 1.05 , which is consistent with the results of Liszt \& Lucas 1998.

\section{RADIATIVE TRANSFER ANALYSIS}

Multiple absorption lines from $\mathrm{CO}$ and $\mathrm{HCO}^{+}$allow us to constrain the physical properties along a sightline. Given the specific input parameters (kinetic temperature, $\mathrm{T}_{\text {kin }}$, volume density of collision partners, $n$ (e.g. $\mathrm{p}-\mathrm{H}_{2}, \quad$ o- $\mathrm{H}_{2}, \mathrm{e}^{-}$), column density of specific molecule $N_{\text {mol }}$, and full width at half maximum (FWHM) of a specific line, $\delta v)$, we are able to model the observed spectra with RADEX (van der Tak et al. 2007).

In this work, we considered $\mathrm{p}-\mathrm{H}_{2}$ and $\mathrm{o}-\mathrm{H}_{2}$ ([o$\left.\mathrm{H}_{2}\right] /\left[\mathrm{p}-\mathrm{H}_{2}\right]=10^{-3}$; Le Bourlot 1991; Dislaire et al. 2012) as the collision partners of $\mathrm{CO}$, and $\mathrm{H}_{2}$ and electrons $\left(\left[\mathrm{e}^{-} / \mathrm{H}_{2}\right]=10^{-4}\right.$; Bhattacharyya et al. 1981; Lucas \& Liszt 1996; Liszt 2012) as the collision partners of $\mathrm{HCO}^{+}$. Adopting a kinetic temperature of $100 \mathrm{~K}$ for the diffuse ISM (Goldsmith 2013; Gerin et al. 2015), we vary the free parameters $\mathrm{n}_{\mathrm{H}_{2}}, \mathrm{~N}_{\mathrm{CO}}$, and $\mathrm{N}_{\mathrm{HCO}}+$ to find the optimum solutions by minimizing $\chi^{2}$ for the four absorption spectra of $\mathrm{CO}$ and $\mathrm{HCO}^{+}$toward $3 \mathrm{C} 454.3$. Here $\chi^{2}$ is defined as

$$
\chi^{2}=\frac{1}{n} \sum_{i=1}^{n} \frac{\left(\tau_{\text {model }}^{i}-\tau_{o b s}^{i}\right)^{2}}{\sigma_{o b s}^{i}{ }^{2}},
$$

where $\tau_{\text {model }}^{\mathrm{i}}$ is the output optical depth generated by RADEX, $\tau_{\mathrm{obs}}^{\mathrm{i}}$ is the observed optical depth of the four spectra, and $\sigma_{\text {obs }}^{\mathrm{i}}$ is the uncertainty of the observed optical depth for each transition.

For the other sources, we have only one transition for $\mathrm{CO}$ and one for $\mathrm{HCO}^{+}$. The same kinetic temperature $(100 \mathrm{~K})$ and the $\mathrm{n}_{\mathrm{H}_{2}}$ obtained for $3 \mathrm{C} 454.3\left(88 \mathrm{~cm}^{-3}\right)$ were then used as the initial assumptions when modeling the optical depth toward the other sources.

Analyzing the fitting results, we obtain the following results:

- The best fit values for $\mathrm{n}_{\mathrm{H}_{2}}, \mathrm{~N}_{\mathrm{CO}}$, and $\mathrm{N}_{\mathrm{HCO}}{ }^{+}$ toward $3 \mathrm{C} 454.3$ are $87 \mathrm{~cm}^{-3}, 3.6 \times 10^{14} \mathrm{~cm}^{-2}$, and $1.8 \times 10^{11} \mathrm{~cm}^{-2}$, respectively. The derived excitation temperature values are $4.1 \mathrm{~K}$ for $\mathrm{CO}$ and $2.7 \mathrm{~K}$ for $\mathrm{HCO}^{+}$. Our results are consistent with Goldsmith $2013\left(\mathrm{~T}_{\text {ex }}(\mathrm{CO}) \sim 2.7-13.6 \mathrm{~K}\right)$ and Godard et al. $2010\left(\mathrm{~T}_{\mathrm{ex}}(\mathrm{CO}) \sim 2.7-3.0 \mathrm{~K}\right)$.

- By varying the assumed $\mathrm{T}_{\text {kin }}$ value from 10 to $300 \mathrm{~K}$, we found that the fractional difference are within $1 \%$ for $\mathrm{T}_{\mathrm{ex}}(\mathrm{CO})$ and $\mathrm{N}_{\mathrm{CO}}$, and $0.1 \%$ for $\mathrm{T}_{\mathrm{ex}}\left(\mathrm{HCO}^{+}\right)$and $\mathrm{N}_{\mathrm{HCO}}{ }^{+}$.

- The column densities of $\mathrm{CO}$ and $\mathrm{HCO}^{+}$components in our sample are in the range of (0.1-19) $\times 10^{14} \mathrm{~cm}^{-2}$ and $(0.07-8.8) \times 10^{11} \mathrm{~cm}^{-2}$, respectively. Three $\sigma$ upper limits in $\mathrm{CO}$ column density are given for those components without $\mathrm{CO}$ (1-0) detections (red points with arrows in Figure 2 ). As shown in Figure 2 (a), the column densities of $\mathrm{CO}$ and $\mathrm{HCO}^{+}$show a positive correlation. The ratio of $\mathrm{N}\left(\mathrm{HCO}^{+}\right) / \mathrm{N}(\mathrm{CO})$ decreases by an order of magnitude as the $\mathrm{CO}$ column density goes from lower to higher, as shown in Figure 2 (b). Similar results have also been reported by Turner 1995, who found that the $\mathrm{N}\left(\mathrm{HCO}^{+}\right) / \mathrm{N}(\mathrm{CO})$ ] ratio is 10 times higher toward regions with low extinction $\left(\mathrm{A}_{\mathrm{v}}<1 \mathrm{mag}\right)$ than that of higher extinction $\left(\mathrm{A}_{\mathrm{v}}>1.5 \mathrm{mag}\right)$. Our samples complement the data points of Lucas \& Liszt (1996), extending the relation to a much lower column density (down to $7 \times 10^{9} \mathrm{~cm}^{-2}$ for $\left.\mathrm{HCO}^{+}\right)$.

\section{5. $\mathrm{HCO}^{+}$AS A MOLECULAR GAS TRACER}

In this section, we derive the relative abundance of $\mathrm{CO}$ and $\mathrm{HCO}^{+}$with respect to $\mathrm{H}_{2}$ by $\mathrm{N}_{\mathrm{H}_{2}}=\frac{\mathrm{N}_{\mathrm{H}}-\mathrm{N}_{\mathrm{HI}}}{2}$. Here, $\mathrm{N}_{\mathrm{H}}$ is estimated from $\mathrm{E}(\mathrm{B}-\mathrm{V})$ (shown in Table 1 ), as described below. $\mathrm{N}_{\mathrm{H}}$ is obtained as described in Section 2, and for the majority of sightlines represents a lower limit under the optically thin assumption.

Interstellar reddening is caused by dust absorption and scattering. Observations from Ly $\alpha$ absorption toward 100 stars showed a linear relation between total gas column density and interstellar reddening: $\mathrm{N}_{\mathrm{H}} / \mathrm{E}(\mathrm{B}-\mathrm{V})=5.8 \times 10^{21} \mathrm{~cm}^{-2} \mathrm{mag}^{-1}$ (Bohlin et al. 1978). The ratio has been widely used to convert reddening to total gas column density. However, different $\mathrm{N}_{\mathrm{H}}\left(\mathrm{cm}^{-2}\right) / \mathrm{E}(\mathrm{B}-\mathrm{V})$ ratios have been found in different environments by recent observations. Planck Collaboration et al. (2014) compared reddening from Sloan Digital Sky Survey (SDSS) measurements of quasars with total gas column density in the diffuse ISM, and found a value $\sim 1.2$ times larger than in Bohlin et al. (1978). Liszt $(2014 a, b)$ compared $\mathrm{N}_{\mathrm{HI}}$ and $\mathrm{E}(\mathrm{B}-\mathrm{V})$, resulting in $\mathrm{N}_{\mathrm{H}}\left(\mathrm{cm}^{-2}\right) / \mathrm{E}(\mathrm{B}-\mathrm{V})=8.3 \times 10^{21} \mathrm{~cm}^{-2} \mathrm{mag}^{-1}$ for $|\mathrm{b}| \geq 20^{\circ}$ and $0.015 \leq \mathrm{E}(\mathrm{B}-\mathrm{V}) \leq 0.075$. Similar results have also been found by Lenz et al. (2017) in low $\mathrm{N}_{\mathrm{H} \mathrm{I}}\left(<4 \times 10^{20} \mathrm{~cm}^{-2}\right)$ regions, and by Nguyen et al. (2018) along purely atomic sightlines $\left(8.8 \times 10^{21}\right.$ and $(9.4 \pm 1.6) \times 10^{21} \mathrm{~cm}^{-2} \mathrm{mag}^{-1}$, respectively). $\gamma$-ray observations from Fermi show the ratio decreases from atomic clouds to dense molecular clouds by a factor of $\sim 0.6$ (Remy et al. 2018). In our case, the $\mathrm{E}(\mathrm{B}-\mathrm{V})$ values along sightlines are in the range of 0.06 to $0.67 \mathrm{mag}\left(\mathrm{A}_{\mathrm{v}}: 0.2 \sim 2 \mathrm{mag}\right)$, including both diffuse, atomic-dominated and translucent molecu- 


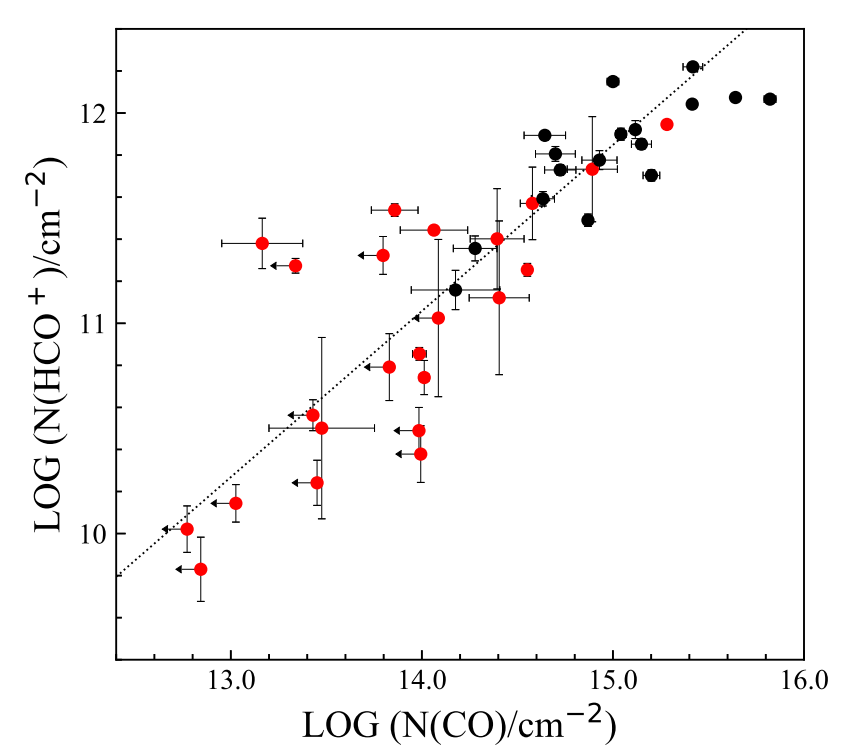

(a)

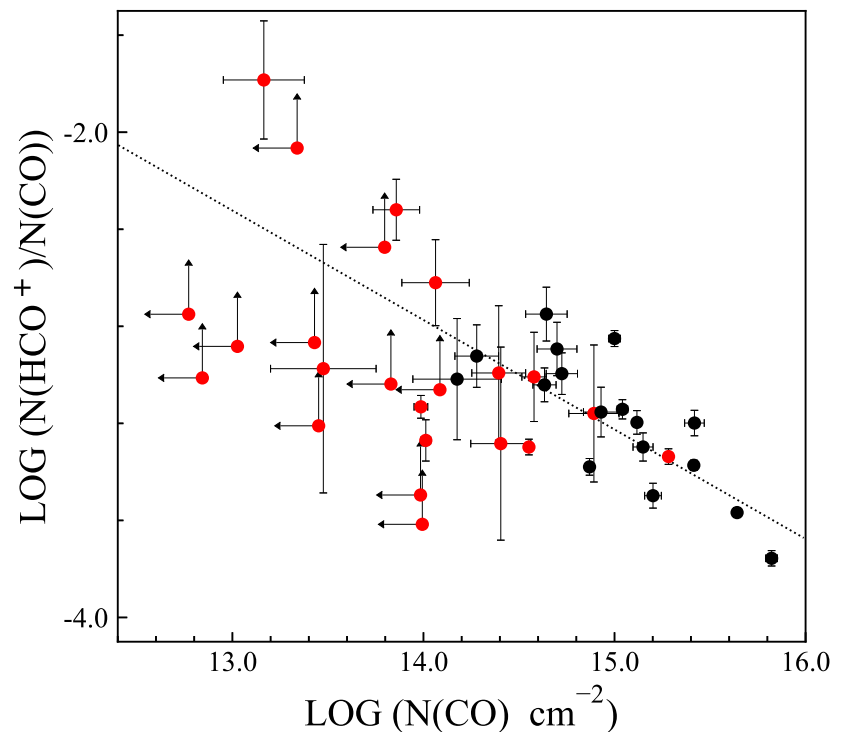

(b)

Figure 2. (a): $\mathrm{HCO}^{+}$column density vs. $\mathrm{CO}$ column density in a log-log scale; (b): $\mathrm{N}\left(\mathrm{HCO}^{+}\right) / \mathrm{N}(\mathrm{CO})$ ratio vs. $\mathrm{CO}$ column density in a log-log scale. The red dots are from our work and black dots are from Liszt \& Lucas (1998, CO column density in Table 5) and Lucas \& Liszt (1996, $\mathrm{HCO}^{+}$column density in Table 2). The black dotted lines show the fit slope to the data points from all components (upper limits are not included).

lar gas environments. We still adopt the value $\mathrm{N}_{\mathrm{H}}\left(\mathrm{cm}^{-2}\right) / \mathrm{E}(\mathrm{B}-\mathrm{V})$ derived by Bohlin et al. 1978 for sightlines with $\mathrm{E}(\mathrm{B}-\mathrm{V})>0.5 \mathrm{mag}$ (1730-130, 1741038, and 1742-078). However, this ratio will lead to $\mathrm{N}_{\mathrm{H}}<\mathrm{N}_{\mathrm{H}}$ t towards atomic dominated sightlines (3C454.3, 3C120, 2148+0657, and 2232+1143). Thus, we adopt the value of $(9.4 \pm 1.6) \times 10^{21} \mathrm{~cm}^{-2} \mathrm{mag}^{-1}$ for sightlines with $\mathrm{E}(\mathrm{B}-\mathrm{V})<0.5 \mathrm{mag}$ (Table 1$)$.

The relation between the abundance of $\mathrm{CO}$ and $\mathrm{HCO}^{+}$ and $\mathrm{H}_{2}$ column density is shown in Figure 3 (datapoints in Lucas \& Liszt 1996; Liszt \& Lucas 1998 are included). The abundance of $\mathrm{CO}$ ranges from $(0.2 \pm 0.1)$ $\times 10^{-6}$ to $(5 \pm 4) \times 10^{-6}$, which is consistent with previous work from far-ultraviolet spectra of the diffuse ISM (mean value of $\sim 3 \times 10^{-6}$ in Burgh et al. 2007). This value is much lower than typical values in dense molecular clouds (e.g., $10^{-5} \sim 10^{-4}$ in Taurus, Goldsmith et al. 2008). The abundance of $\mathrm{HCO}^{+}$ranges from $(1.0 \pm 0.4) \times 10^{-9}$ to $(3 \pm 2) \times 10^{-9}$. The mean values of molecular abundance are $(2.1 \pm 1.1) \times 10^{-6}$ for $\mathrm{CO}$ and $(1.7 \pm 0.3) \times 10^{-9}$ for $\mathrm{HCO}^{+}$(upper limits are not included). The Pearson correlation coefficient values between the molecular column density and $\mathrm{N}_{\mathrm{H}_{2}}$ are 0.33 for $\mathrm{CO}$ and 0.93 for $\mathrm{HCO}^{+}$, indicating that $\mathrm{HCO}^{+}$ is a better tracer of molecular gas than $\mathrm{CO}$.

\section{THE CO X-FACTOR}

The integrated intensity of $\mathrm{CO}$ emission $\left(\mathrm{W}_{\mathrm{CO}}\right)$ is considered to be a good tracer of $\mathrm{H}_{2}$ column density. The CO-to- $\mathrm{H}_{2}$ conversion factor $\left(\mathrm{X}_{\mathrm{CO}}\right)$, which is defined as $\mathrm{X}_{\mathrm{CO}}=\mathrm{N}_{\mathrm{H}_{2}} / \mathrm{W}_{\mathrm{CO}}$, is important for understanding the evolution of star formation, and especially for external galaxies. Due to the distance of extra-galactic objects, $\mathrm{CO}$ may be the primary or the only molecular tracer available to explore star formation and determine the total gas mass.

We have reconstructed the CO integrated intensity from the $\tau$ and $\mathrm{T}_{\text {ex }}$ obtained from our RADEX modeling. The relation between $\mathrm{W}_{\mathrm{CO}}$ and $\mathrm{N}_{\mathrm{H}_{2}}$ is shown in Figure 4. The average $\mathrm{X}$-factor we obtain in our sightlines is $(14 \pm 3) \times 10^{20} \mathrm{~cm}^{-2}\left(\mathrm{~K} \mathrm{~km} \mathrm{~s}^{-1}\right)^{-1}$, which is 6 times larger than the average value in the Milky Way (Bolatto et al. 2013). Our results are in agreement with observations in regions without ${ }^{12} \mathrm{CO}$ and ${ }^{13} \mathrm{CO}$ emission detections in Taurus $\left(1.2 \times 10^{21} \mathrm{~cm}^{-2}\right.$ $\left(\mathrm{K} \mathrm{km} \mathrm{s}^{-1}\right)^{-1}$ in Pineda et al. 2010). Numerical simulations by Shetty et al. 2011 presented $\mathrm{X}_{\mathrm{CO}}-\mathrm{N}_{\mathrm{H}_{2}}$ relations in molecular clouds with different conditions (e.g., density, metallicity), and they found a high $\mathrm{X}$-factor $\left(\sim 10^{21} \mathrm{~cm}^{-2}\left(\mathrm{~K} \mathrm{~km} \mathrm{~s}^{-1}\right)^{-1}\right)$ where $\mathrm{CO}$ abundance is low $\left(10^{-6}\right)$. In addition, numerical simulations and observations also suggest that turbulence, visual extinction, and metallicity will all influence the CO X-factor (Bell et al. 2006; Shetty et al. 2011). This result indi- 
Table 1. The $\mathrm{E}(\mathrm{B}-\mathrm{V}), \mathrm{N}_{\mathrm{H}}, \mathrm{N}_{\mathrm{HI}}, \mathrm{N}_{\mathrm{H}_{2}}$, atomic fraction, abundance of $\mathrm{CO}$ and $\mathrm{HCO}^{+}$and rms towards 8 sources

\begin{tabular}{|c|c|c|c|c|c|c|c|c|c|}
\hline \multirow[t]{2}{*}{ Source } & \multirow{2}{*}{$\begin{array}{c}\mathrm{E}(\mathrm{B}-\mathrm{V}) \\
\mathrm{mag}\end{array}$} & \multirow{2}{*}{$\begin{array}{c}\mathrm{N}_{\mathrm{H}} \\
10^{21} \mathrm{~cm}^{-2}\end{array}$} & \multirow{2}{*}{$\begin{array}{c}\mathrm{N}_{\mathrm{H} \mathrm{I}} \\
10^{20} \mathrm{~cm}^{-2}\end{array}$} & \multirow{2}{*}{$\begin{array}{c}\mathrm{N}_{\mathrm{H}_{2}} / 10^{20} \mathrm{~cm}^{-2} \\
10^{20} \mathrm{~cm}^{-2}\end{array}$} & \multirow[t]{2}{*}{$\mathrm{f}_{\text {atomic }}$} & \multirow{2}{*}{$\begin{array}{c}\mathrm{f}_{\mathrm{CO}} \\
10^{-6}\end{array}$} & \multirow{2}{*}{$\begin{array}{c}\mathrm{f}_{\mathrm{HCO}}+ \\
10^{-9}\end{array}$} & \multicolumn{2}{|c|}{$\mathrm{rms}$} \\
\hline & & & & & & & & $\tau_{\mathrm{CO}}$ & $\tau_{\mathrm{HCO}^{+}}$ \\
\hline $3 \mathrm{C} 454.3$ & $0.106 \pm 0.002$ & $1.0 \pm 0.2^{\mathrm{a}}$ & $7.7 \pm 0.1^{\mathrm{c}}$ & $1.1 \pm 0.9$ & $0.7 \pm 0.1$ & $5 \pm 4$ & $3 \pm 2$ & 0.003 & 0.001 \\
\hline $3 \mathrm{C} 120$ & $0.265 \pm 0.006$ & $2.5 \pm 0.4^{\mathrm{a}}$ & $18.2 \pm 0.1^{\mathrm{c}}$ & $3 \pm 2$ & $0.7 \pm 0.1$ & $0.5 \pm 0.3$ & $1.0 \pm 0.6$ & 0.02 & 0.004 \\
\hline $0607-157$ & $0.203 \pm 0.004$ & $1.9 \pm 0.3^{\mathrm{a}}$ & $11.3 \pm 0.2$ & $4 \pm 2$ & $0.6 \pm 0.1$ & $0.2 \pm 0.1$ & $1.0 \pm 0.4$ & 0.01 & 0.009 \\
\hline $1730-130$ & $0.513 \pm 0.009$ & $2.98 \pm 0.05^{\mathrm{b}}$ & $16.6 \pm 0.3$ & $6.6 \pm 0.3$ & $0.56 \pm 0.01$ & $2.9 \pm 0.2$ & $1.7 \pm 0.1$ & 0.008 & 0.01 \\
\hline $1741-038$ & $0.530 \pm 0.008$ & $3.07 \pm 0.05^{\mathrm{b}}$ & $17.6 \pm 0.4$ & $6.6 \pm 0.3$ & $0.57 \pm 0.01$ & $1.0 \pm 0.2$ & $1.2 \pm 0.3$ & 0.01 & 0.02 \\
\hline $1742-078$ & $0.672 \pm 0.008$ & $3.90 \pm 0.05^{\mathrm{b}}$ & $22.7 \pm 0.5$ & $8.1 \pm 0.3$ & $0.58 \pm 0.01$ & $1.3 \pm 0.3$ & $1.1 \pm 0.4$ & 0.05 & 0.04 \\
\hline $2148+0657$ & $0.062 \pm 0.002$ & $0.6 \pm 0.1^{\mathrm{a}}$ & $4.3 \pm 0.1$ & $0.8 \pm 0.5$ & $0.7 \pm 0.1$ & $<0.3$ & $3 \pm 2$ & 0.006 & 0.008 \\
\hline $2232+1143$ & $0.062 \pm 0.002$ & $0.6 \pm 0.1^{\mathrm{a}}$ & $4.8 \pm 0.1$ & $0.53 \pm 0.51$ & $0.8 \pm 0.1$ & $<1.0$ & $1.3 \pm 1.2$ & 0.002 & 0.0008 \\
\hline
\end{tabular}

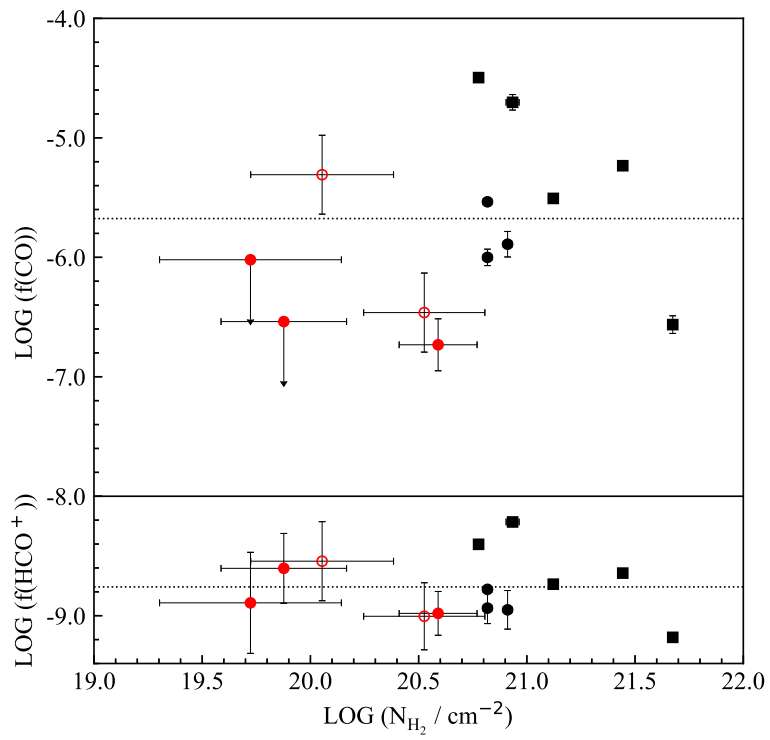

Figure 3. The relation between the abundance of $\mathrm{CO}$, $\mathrm{HCO}^{+}$and $\mathrm{N}_{\mathrm{H}_{2}}$ (circles from our work, squares from Lucas \& Liszt 1996; Liszt \& Lucas 1998). Red and black points denote sightlines using $\mathrm{N}_{\mathrm{H}} / \mathrm{E}(\mathrm{B}-\mathrm{V})$ conversion factor of Nguyen et al. 2018 and Bohlin et al. 1978, respectively. Empty red circles denote sightlines with H I opacity correction. The black dotted lines show the mean values of molecular abundance (points with upper limits are not included).

cates that the $\mathrm{X}$-factor should be handled carefully in different physical environments, otherwise the $\mathrm{H}_{2}$ column density may be underestimated in diffuse gas.

\section{CONCLUSION}

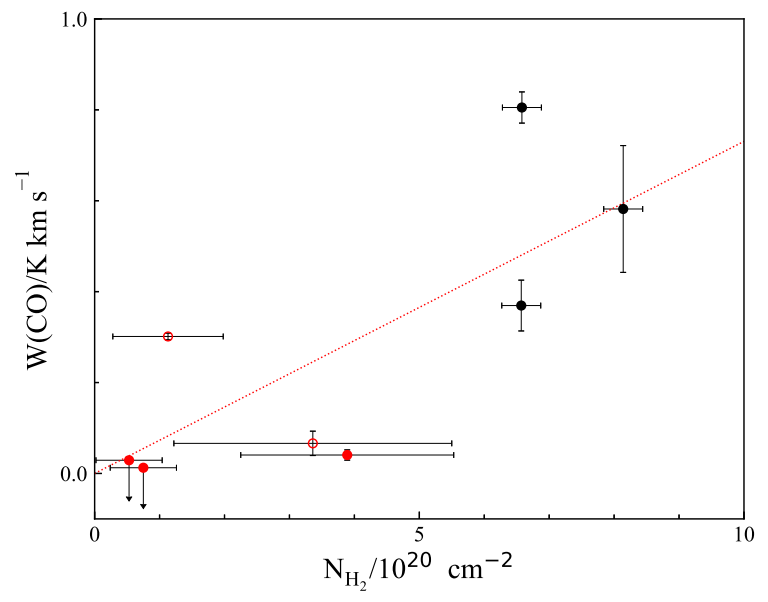

Figure 4. The relation of the $\mathrm{H}_{2}$ column density and the integrated intensity of $\mathrm{CO}(1-0)$. Red and black points denote sightlines using $\mathrm{N}_{\mathrm{H}} / \mathrm{E}(\mathrm{B}-\mathrm{V})$ conversion factor of Nguyen et al. 2018 and Bohlin et al. 1978, respectively. Empty red circles denote sightlines with H I opacity correction. The red dotted line shows the fit results corresponding to X-factor (points with upper limit are not included).

We have performed ALMA observations toward 13 continuum sources. The unprecedented sensitivity provided by ALMA allows us to probe cold molecular gas down to a $\tau_{\mathrm{RMS}}^{\mathrm{CO}}$ of 0.002 and a $\tau_{\mathrm{RMS}}^{\mathrm{HCO}^{+}}$of 0.0008 . Absorption in both the $\mathrm{CO}(1-0)$ and $\mathrm{HCO}^{+}(1-0)$ lines were detected toward 6 of 13 sources. In addition, $\mathrm{HCO}^{+}$absorption was detected toward two bandpass calibrators $(2148+0657$ and $2232+1143)$. Two higher transitions of $\mathrm{CO}(2-1)$ and $\mathrm{HCO}^{+}(3-2)$ were observed and detected towards $3 \mathrm{C} 454.3$. The main conclusions are as follows: 
1. Twelve CO (1-0) components and twenty-three $\mathrm{HCO}^{+}(1-0)$ components are detected, revealing $\mathrm{CO}$ in diffuse gas with extinction as low as $A_{v} \sim 0.32 \mathrm{mag}$, where no $\mathrm{CO}$ emission was detected.

2. By modeling multiple absorption lines - CO (1-0) and (2-1), and $\mathrm{HCO}^{+}(1-0)$ and (3-2) - toward 3C454.3, we derived excitation temperatures of $4.1 \mathrm{~K}$ for $\mathrm{CO}$ and $2.7 \mathrm{~K}$ for $\mathrm{HCO}^{+}$. Those temperatures are comparable to the continuum background temperature, making them extremely hard to detect in emission surveys.

3. The derived column densities of $\mathrm{CO}$ and $\mathrm{HCO}^{+}$ show a positive correlation, while the abundance ratio $[\mathrm{CO}] /\left[\mathrm{HCO}^{+}\right]$increases by an order of magnitude as the $\mathrm{CO}$ column density goes from lower to higher. $\mathrm{N}_{\mathrm{HCO}}{ }^{+}$ has tight linear relation with $\mathrm{N}_{\mathrm{H}_{2}}$ while $\mathrm{N}_{\mathrm{CO}}$ does not. Two sightlines without $\mathrm{CO}$ detection are found to be rich in $\mathrm{HCO}^{+}$. All facts are consistent with the understanding that, in this intermediate extinction region, $\mathrm{HCO}^{+}$is a better tracer of total molecular gas than CO.

4. Toward these absorption sight-lines, we derived the CO X-factor to be $(14 \pm 3) \times 10^{20} \mathrm{~cm}^{-2}\left(\mathrm{~K} \mathrm{~km} \mathrm{~s}^{-1}\right)^{-1}$, 6 times larger than the typical value found in the Milky Way. This result is consistent with simulations of clouds going through $\mathrm{HI}-\mathrm{H}_{2}$ transitions, where the $\mathrm{CO}$ is still building up abundance and has not yet locked up most of the carbon in the ISM.
This work has been supported by the National Natural Science Foundation of China No. 11988101, No. 11725313, No. 11803051, National Key R\&D Program of China No. 2017YFA0402600, the CAS Strategic Priority Research Program No. XDB23000000, and the CAS International Partnership Program No. 114A11KYSB20160008. N.Y. Tang acknowledges the support from the CAS "Light of West China" Program and Young Researcher Grant of National Astronomical Observatories, Chinese Academy of Sciences. J. R. Dawson acknowledges the support of an Australian Research Council (ARC) DECRA Fellowship (project number DE170101086). L. Bronfman and Ricardo Finger acknowledge support from CONICYT project Basal AFB-170002.

This paper makes use of the following ALMA data: ADS/JAO.ALMA\#2015.1.00503.S. ALMA is a partnership of ESO (representing its member states), NSF (USA) and NINS (Japan), together with NRC (Canada) and NSC and ASIAA (Taiwan) and KASI (Republic of Korea), in cooperation with the Republic of Chile. The Joint ALMA Observatory is operated by ESO, AUI/NRAO and NAOJ.

Software: CASA (McMullin et al. 2007), RADEX (van der Tak et al. 2007),

\section{REFERENCES}

Bell, T. A., Roueff, E., Viti, S., \& Williams, D. A. 2006, MNRAS, 371, 1865,

doi: 10.1111/j.1365-2966.2006.10817.x

Bhattacharyya, S. S., Bhattacharyya, B., \& Narayan, M. V. 1981, ApJ, 247, 936, doi: 10.1086/159102

Bohlin, R. C., Savage, B. D., \& Drake, J. F. 1978, ApJ, 224, 132, doi: 10.1086/156357

Bolatto, A. D., Wolfire, M., \& Leroy, A. K. 2013, ARA\&A, 51, 207, doi: 10.1146/annurev-astro-082812-140944

Burgh, E. B., France, K., \& McCandliss, S. R. 2007, ApJ, 658, 446, doi: 10.1086/511259

Dame, T. M., Hartmann, D., \& Thaddeus, P. 2001, ApJ, 547, 792, doi: 10.1086/318388

Dickey, J. M. 2013, in IAU Symposium, Vol. 288, Astrophysics from Antarctica, ed. M. G. Burton, X. Cui, \& N. F. H. Tothill, 135-138

Dislaire, V., Hily-Blant, P., Faure, A., et al. 2012, A\&A, 537, A20, doi: 10.1051/0004-6361/201117765

Draine, B. T. 2011, Physics of the Interstellar and Intergalactic Medium

Gerin, M., Ruaud, M., Goicoechea, J. R., et al. 2015, A\&A, 573, A30, doi: 10.1051/0004-6361/201424349
Godard, B., Falgarone, E., Gerin, M., Hily-Blant, P., \& de Luca, M. 2010, A\&A, 520, A20, doi: 10.1051/0004-6361/201014283

Goldsmith, P. F. 2013, ApJ, 774, 134, doi: $10.1088 / 0004-637 \mathrm{X} / 774 / 2 / 134$

Goldsmith, P. F., Heyer, M., Narayanan, G., et al. 2008, ApJ, 680, 428, doi: 10.1086/587166

Green, G. M., Schlafly, E. F., Zucker, C., Speagle, J. S., \& Finkbeiner, D. P. 2019, arXiv e-prints, arXiv:1905.02734. https://arxiv.org/abs/1905.02734

Green, G. M., Schlafly, E. F., Finkbeiner, D., et al. 2018, MNRAS, 478, 651, doi: 10.1093/mnras/sty 1008

Grenier, I. A., Casandjian, J.-M., \& Terrier, R. 2005, Science, 307, 1292, doi: 10.1126/science.1106924

Heiles, C., \& Troland, T. H. 2003, ApJS, 145, 329, doi: $10.1086 / 367785$

Kalberla, P. M. W., \& Haud, U. 2015, A\&A, 578, A78, doi: 10.1051/0004-6361/201525859

Kalberla, P. M. W., McClure-Griffiths, N. M., Pisano, D. J., et al. 2010, A\&A, 521, A17, doi: 10.1051/0004-6361/200913979 
Langer, W. D., Velusamy, T., Pineda, J. L., Willacy, K., \& Goldsmith, P. F. 2014, A\&A, 561, A122, doi: $10.1051 / 0004-6361 / 201322406$

Le Bourlot, J. 1991, A\&A, 242, 235

Lenz, D., Hensley, B. S., \& Doré, O. 2017, ApJ, 846, 38, doi: $10.3847 / 1538-4357 /$ aa84af

Li, D., Tang, N., Nguyen, H., et al. 2018, ApJS, 235, 1, doi: $10.3847 / 1538-4365 /$ aaa762

Liszt, H. 2014a, ApJ, 780, 10, doi: $10.1088 / 0004-637 \mathrm{X} / 780 / 1 / 10$

—. 2014b, ApJ, 783, 17, doi: 10.1088/0004-637X/783/1/17

Liszt, H. S. 2012, A\&A, 538, A27, doi: 10.1051/0004-6361/201117882

Liszt, H. S., \& Lucas, R. 1998, A\&A, 339, 561

Liszt, H. S., Pety, J., \& Lucas, R. 2010, A\&A, 518, A45, doi: $10.1051 / 0004-6361 / 201014510$

Lucas, R., \& Liszt, H. 1996, A\&A, 307, 237

McClure-Griffiths, N. M., Pisano, D. J., Calabretta, M. R., et al. 2009, ApJS, 181, 398, doi: $10.1088 / 0067-0049 / 181 / 2 / 398$

McMullin, J. P., Waters, B., Schiebel, D., Young, W., \& Golap, K. 2007, in Astronomical Society of the Pacific Conference Series, Vol. 376, Astronomical Data Analysis Software and Systems XVI, ed. R. A. Shaw, F. Hill, \& D. J. Bell, 127

Nguyen, H., Dawson, J. R., Miville-Deschênes, M.-A., et al. 2018, ApJ, 862, 49, doi: 10.3847/1538-4357/aac82b

Pineda, J. L., Goldsmith, P. F., Chapman, N., et al. 2010, ApJ, 721, 686, doi: 10.1088/0004-637X/721/1/686

Pineda, J. L., Langer, W. D., Velusamy, T., \& Goldsmith, P. F. 2013, A\&A, 554, A103, doi: $10.1051 / 0004-6361 / 201321188$
Planck Collaboration, Ade, P. A. R., Aghanim, N., et al. 2011, A\&A, 536, A19, doi: 10.1051/0004-6361/201116479

Planck Collaboration, Abergel, A., Ade, P. A. R., et al. 2014, A\&A, 571, A11, doi: $10.1051 / 0004-6361 / 201323195$

Remy, Q., Grenier, I. A., Marshall, D. J., \& Casand jian, J. M. 2018, A\&A, 616, A71, doi: $10.1051 / 0004-6361 / 201731488$

Remy, Q., Grenier, I. A., Marshall, D. J., \& Casandjian, J. M. 2017, A\&A, 601, A78, doi: $10.1051 / 0004-6361 / 201629632$

Riquelme, D., Bronfman, L., Mauersberger, R., et al. 2018, A\&A, 610, A43, doi: 10.1051/0004-6361/201730602

Shetty, R., Glover, S. C., Dullemond, C. P., et al. 2011, MNRAS, 415, 3253, doi: $10.1111 / j .1365-2966.2011 .18937 . x$

Turner, B. E. 1995, ApJ, 449, 635, doi: 10.1086/176085 van der Tak, F. F. S., Black, J. H., Schöier, F. L., Jansen, D. J., \& van Dishoeck, E. F. 2007, A\&A, 468, 627, doi: $10.1051 / 0004-6361: 20066820$

van Dishoeck, E. F., \& Black, J. H. 1988, ApJ, 334, 771, doi: $10.1086 / 166877$

Wilson, R. W., Jefferts, K. B., \& Penzias, A. A. 1970, ApJL, 161, L43, doi: 10.1086/180567

Winkel, B., Kerp, J., Flöer, L., et al. 2016, A\&A, 585, A41, doi: 10.1051/0004-6361/201527007

Wolfire, M. G., Hollenbach, D., \& McKee, C. F. 2010, ApJ, 716, 1191, doi: 10.1088/0004-637X/716/2/1191 